(CPP and PPP), characteristic impedance $\left(\mathrm{Z}_{\mathrm{c}}\right)$, augmentation index, and forward pressure wave. Race-specific characteristic means and proportions were tested using Student's T- or Chi-square tests. After further sex stratification, significant variation in characteristics among sex within race were tested using nested ANOVA. RESULTS/ ANTICIPATED RESULTS: Characteristics of the study group stratified by race were found to be similar. Vascular measures stratified by race revealed blacks to have significantly higher $Z_{c}(p=0.03)$ and PPP $(p=0.03)$ than whites. DISCUSSION/SIGNIFICANCE OF IMPACT: In this study of vascular hemodynamics in young black and white participants we found differences in $Z_{c}$ and PPP. Findings suggest that differing relations between proximal aortic diameter and wall stiffness may contribute to the racial disparity in CVE in adults. This finding could offer an explanation to the beneficial effects of treatment modalities that target aortic stiffness in blacks.

4439

\section{Comparing the Impact of Adding an Educational Video Presentation to Universal Self-Consent for Remnant Clinical Biospecimens: A Single Blind Randomized- Control Trial}

Andrew Kyle ${ }^{1}$, Stephanie E. Soares ${ }^{1}$, Machelle D. Wilson ${ }^{1}$, Nicholas R. Anderson ${ }^{1}$, and Javier E. Lopez ${ }^{1}$

${ }^{1}$ University of California, Davis

OBJECTIVES/GOALS: BURRITO is an efficient strategy that provides full disclosure in the electronic medical record of a patient's preference in real time. BURRITO uses printed materials only to inform patients and has a $<50 \%$ rates of consent. We hypothesized that adding an informational video to the printed materials would increase donations. METHODS/STUDY POPULATION: This study was IRB-approved and was considered minimal risk. The BURRITO self-consent workflow process (Soares et. al, Biopreservation and Biobanking, IN PRINT) was developed in an outpatient cardiology clinic. In the same clinic, patients were randomized to receiving printed materials only (standard procedure) or the printed materials plus a 2.5-minute informational video (intervention) while waiting for the physician in the exam room. Randomization occurred at the level of the day in clinic. Patients were blinded to the nature of the study. Following the presentation of information, the patient's decision on consent for donation was documented in the electronic record by ancillary clinical staff. Rates of consent were analyzed by a statistician not involved in the experiment and after completion of trial. RESULTS/ANTICIPATED RESULTS: Thirty-five clinic days were randomized to either intervention (17 days) or standard (18 days), and a total of 255 patients decided during their visit to either "opt-in" or "opt-out" to donating remnant biospecimens for future research. One hundred patients opted to defer deciding (28\%). No significant demographic differences were noted between the study arms. The rate of consent was $73 \%$ vs. $58 \%$ in the intervention group and the control group, respectively ( $\mathrm{p}$-value $=0.014)$. This represents an increase in the odds of consenting with an informational video by $96 \%(\mathrm{OR}=1.96,95 \% \mathrm{CI}=1.15$ to 3.34$)$. DISCUSSION/SIGNIFICANCE OF IMPACT: This is the first randomized trial to show that an informational video with printed materials is superior for when patients are self-consenting to opt-in for clinical remnant biospecimen donation. This result adds to the evidence that the BURRITO process plus video (BURRITOv) is an effective approach for biospecimen universal consenting.
Consistent Differences in Lumbar Spine Alignment Between Low Back Pain Subgroups and Sexes during Clinical and Functional Sitting Tests*

Quenten L Hooker ${ }^{1}$, Vanessa M. Lanier ${ }^{1}$, and Linda R. Van Dillen ${ }^{1}$

${ }^{1}$ Washington University in St. Louis

OBJECTIVES/GOALS: Test the validity of a system for subgrouping people with CLBP by comparing lumbar spine alignment in two CLBP subgroups and sexes during clinical tests of maximum flexed and extended sitting and a functional test of preferred sitting. METHODS/STUDY POPULATION: Using the Movement System Impairment classification system, 154 participants with CLBP were subgrouped based on the predominant direction of altered movement and alignment patterns and symptoms during a standardized examination. Participants performed a functional test of preferred sitting followed by clinical tests of maximum flexed and extended sitting in random order. Reflective markers were place superficial to T12, L3 and S1 spinous processes. 3D marker co-ordinate data were collected using an 8 camera motion capture system. Sagittal plane lumbar curvature angle (LCA), defined as the angular distance between T12, L3, and S1 landmarks was calculated for each test. A three-way mixed effect ANOVA model was used to examine the following effects: test, subgroup, sex, test*subgroup, test*sex, subgroup*sex. RESULTS/ANTICIPATED RESULTS: Test: Lumbar alignment patterns were different for flexed $\left.\overline{[\mathrm{LCA}}=7.4^{\circ}(6.1,8.7)\right]$, extended $\left[\mathrm{LCA}=-22.6^{\circ}(-23.9,-21.3)\right]$, and preferred $\left[\mathrm{LCA}=-3.8^{\circ}(-5.2,-2.5)\right]$ sitting tests. LBP subgroup: Rotation-extension $\left[\mathrm{LCA}=-7.5^{\circ}(-8.7,-6.3)\right]$ had more extended lumbar alignment than rotation $\left[\mathrm{LCA}=-5.2^{\circ}(-6.2,-4.2)\right]$. Sex: Women had more extended lumbar alignment [LCA $=-10.3^{\circ}$ $(-11.2,-9.3)]$ than men $\left[\mathrm{LCA}=-2.5^{\circ}(-3.7,-1.2)\right]$. Test* ${ }^{*}$ sex: The difference in lumbar alignment between women and men was smaller during the flexed sitting test [women $=4.2^{\circ}(2.5,5.9)$, men $\left.=9.9^{\circ}(7.8,12.1)\right]$, compared to extended [women $=-27.5^{\circ}$ $(-29.2,-25.8)$, men $=-17.0^{\circ}(-9.2,-14.8)$ ] and preferred [women $=-7.4^{\circ}(-9.1,-5.8)$, men $\left.=-0.3^{\circ}(-2.5,1.8)\right]$. The test $*$ subgroup $(\mathrm{p}=0.84)$ and subgroup*sex $(\mathrm{p}=0.87)$ interactions were not significant.

4491

\section{Cumulative Childhood Trauma Load Across Race in Individuals with Alcohol Use Disorder}

Nia Byrd ${ }^{1}$, Bethany L. Stangl, Melanie L. Schwandt, Nancy

Diazgranados, and Vijay A. Ramchandani

${ }^{1}$ National Institutes of Health

OBJECTIVES/GOALS: Our objective was to investigate racial differences in experiencing multiple categories of childhood trauma (CT) and the differential impact on alcohol use in individuals with alcohol use disorder (AUD). We hypothesized that there would be a differential additive effect of CT categories endorsed and drinking behaviors between racial groups. METHODS/STUDY POPULATION: Participants were recruited through the NIAAA screening protocol where they completed alcohol-related assessments including a 90-day Timeline Followback (TLFB) and the Alcohol Use Disorder Identification Test (AUDIT). Structured Clinical Interviews for DSM disorders were conducted to identify participants with lifetime alcohol dependence (DSM-IV) or AUD (DSM-5) $(\mathrm{N}=1152)$. 